\title{
Amyand's hernia: A case report and review of the literature
}

\author{
Youssef Shaban, Adel Elkbuli*, Mark McKenney, Dessy Boneva \\ Department of Surgery, Kendall Regional Medical Center, 11750 Bird Road, Miami, FL, 33175, United States
}

\section{A R T I C L E I N F O}

\section{Article history:}

Received 10 April 2018

Accepted 29 April 2018

Available online 7 May 2018

\section{Keywords:}

Acute appendicitis

Appendix in hernia

Appendectomy

Amyand hernia

Hernia repair

\begin{abstract}
A B S T R A C $T$
INTRODUCTION: An Amyand hernia is a rare disease where the appendix is found within an inguinal hernia sac. This rare entity is named after the French born English surgeon, Dr. Claudius Amyand. Inguinal hernias are one of the most common surgeries that a general surgeon performs with more than 20 million inguinal hernia repairs performed yearly worldwide. The incidence of finding an appendix within the hernia sac is rare, occurring in less than $1 \%$ of inguinal hernia patients and when complications arise such as inflammation, perforation, or abscess formation it becomes exceptionally rare with an incidence of about $0.1 \%$.

PRESENTATION OF CASE: A 59-year-old male with a history of a previously reducible right inguinal hernia presented to the Emergency Department with acute abdominal pain, right groin mass. Computed tomography (CT) confirmed a right incarcerated inguinal hernia with herniated loops of bowel within the right inguinal region. Patient was subsequently treated with an appendectomy and tension free hernia repair with mesh with a successful outcome.

DISCUSSION: The current generally accepted treatment algorithm for Amyand's hernia is essentially contingent on the appendix's condition within the hernia sac. Controversy exists regarding the application of mesh in type 2 Amyand's hernia. More research is needed to provide surgeons with evidence-based standardized approaches for dealing with this unique situation.

CONCLUSION: This case report reviews a rare entity known as an Amyand's hernia that presented as an incarcerated hernia that was diagnosed intraoperatively with an inflamed appendix, recognized as a type 2 Amyand's hernia.

(C) 2018 The Author(s). Published by Elsevier Ltd on behalf of IJS Publishing Group Ltd. This is an open access article under the CC BY-NC-ND license (http://creativecommons.org/licenses/by-nc-nd/4.0/).
\end{abstract}

\section{Introduction}

In 1735, Dr. Claudius Amyand performed the world's first successful appendectomy, at St. George's Hospital in London. The patient was an 11-year old boy who had an inguinal hernia combined with an acutely inflamed appendix [1]. This rare pathology of an appendix with or without inflammation within the hernia sac is named after the French born English surgeon. Inguinal hernias are one of the most common surgeries that a general surgeon performs with more than 20 million inguinal hernia repairs performed yearly worldwide. The incidence of finding an appendix within the hernia sac is rare, occurring in less than $1 \%$ of inguinal hernia patients and when complications arise such as inflammation, perforation, or abscess formation it becomes exceptionally rare with an incidence of about $0.1 \%$ [2]. With signs and symptoms identical to an incarcerated inguinal hernia, an Amyand's hernia is very difficult to diagnose preoperatively and therefore diagnosis is predominantly made intraoperatively.

\footnotetext{
* Corresponding author.

E-mail addresses: Adel.Elkbulli@HCAHealthcare.com, a.elkbuli@med.miami.edu (A. Elkbuli).
}

Critical to successful outcomes is the correct surgical treatment plan that is predominately made intraoperatively. Therefore, surgeons should be well versed on the different types of Amyand's hernia and the indications for subsequent surgical treatment. There are several factors that dictate appropriate surgical treatment which include appendix condition, characteristics of the hernia, the patient's comorbidities, and other circumstances not well identified due to the lack of research available for this rare disease. Due to the rarity of this disease and a lack of randomized controlled studies there are no evidence-based standardized approaches for dealing with this unique entity. Furthermore, controversy remains regarding whether to perform an appendectomy for a normal appearing appendix or whether mesh should be used for the hernia repair if an appendectomy is performed. Without more research, surgeons may make sub-optimal decisions, which potentially increases patient morbidity.

We present a case report of this rare entity known as an Amyand's hernia that presented as an incarcerated hernia that was diagnosed intraoperatively with an inflamed non-perforated appendix, known as type 2 Amyand's hernia that was subsequently treated with an appendectomy and tension free repair with mesh and we review current literature regarding the management. This case has been reported in line with the SCARE criteria [3]. 


\section{CASE REPORT - OPEN ACCESS}

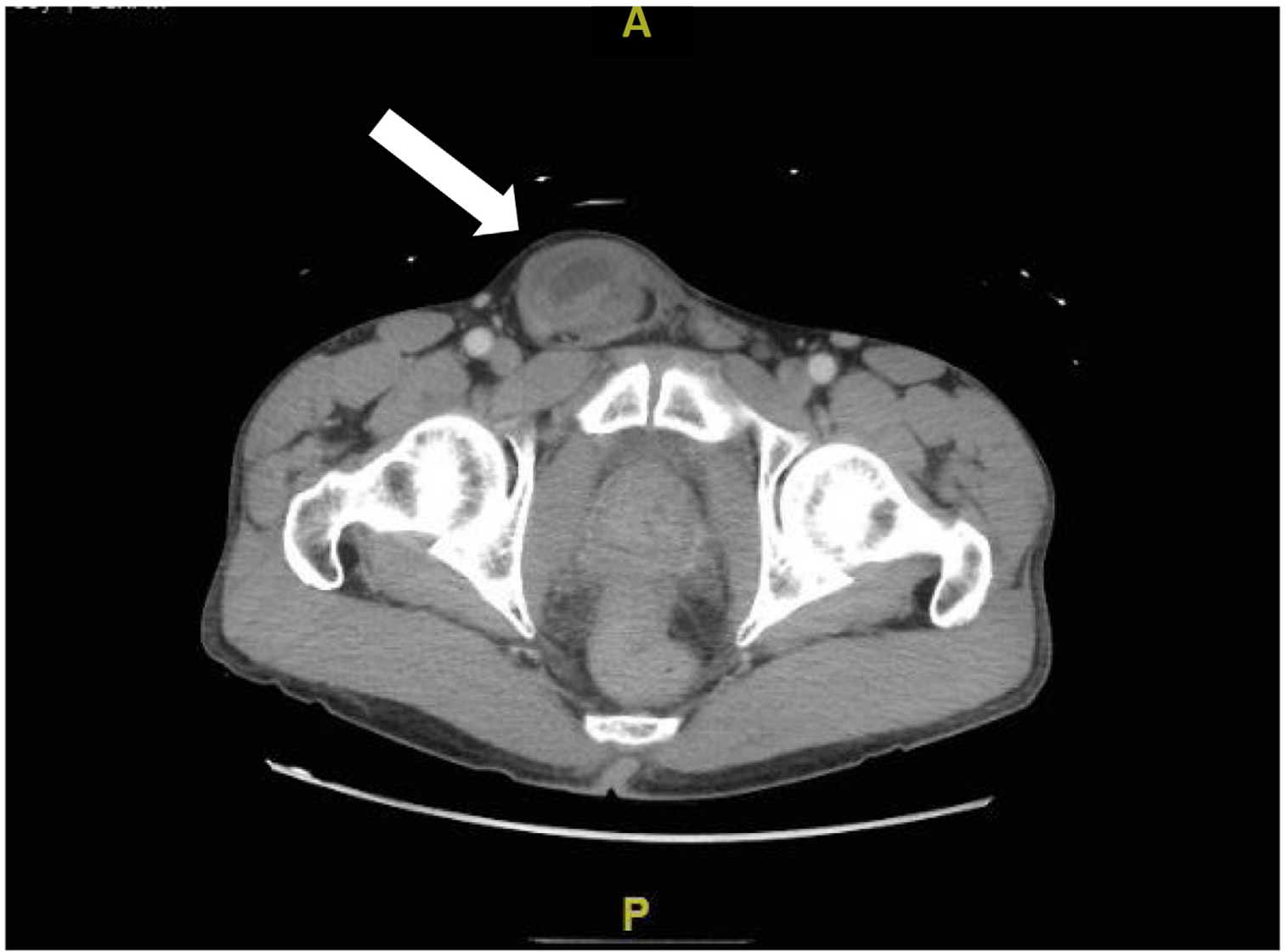

Fig. 1. CT showing an incarcerated right inguinal hernia with small bowel in the right groin/hernia sac.

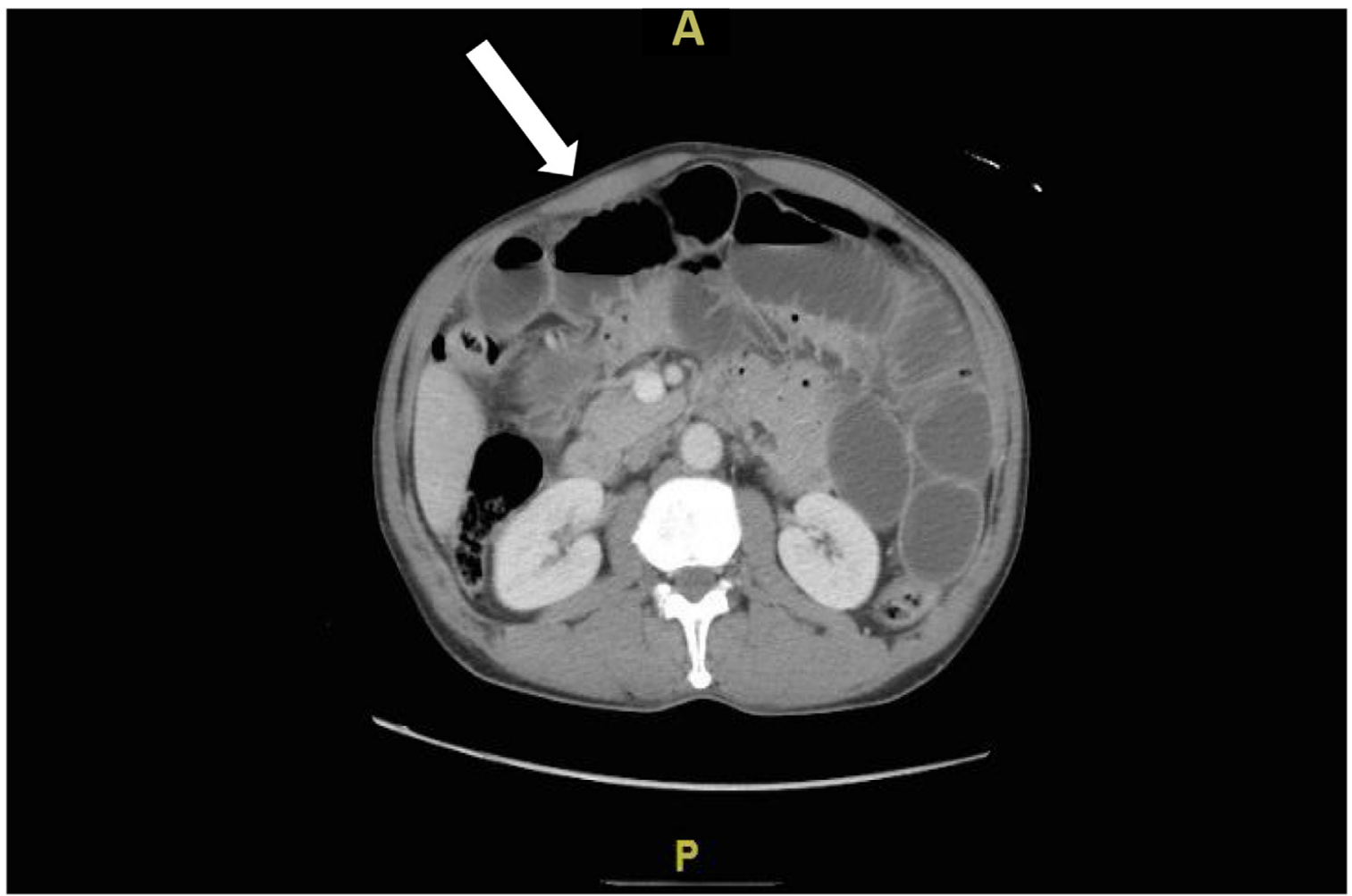

Fig. 2. CT showing small bowel obstruction caused by the incarcerated right inguinal hernia, with fluid filled and dilated loops of small bowel. 


\section{CASE REPORT - OPEN ACCESS}

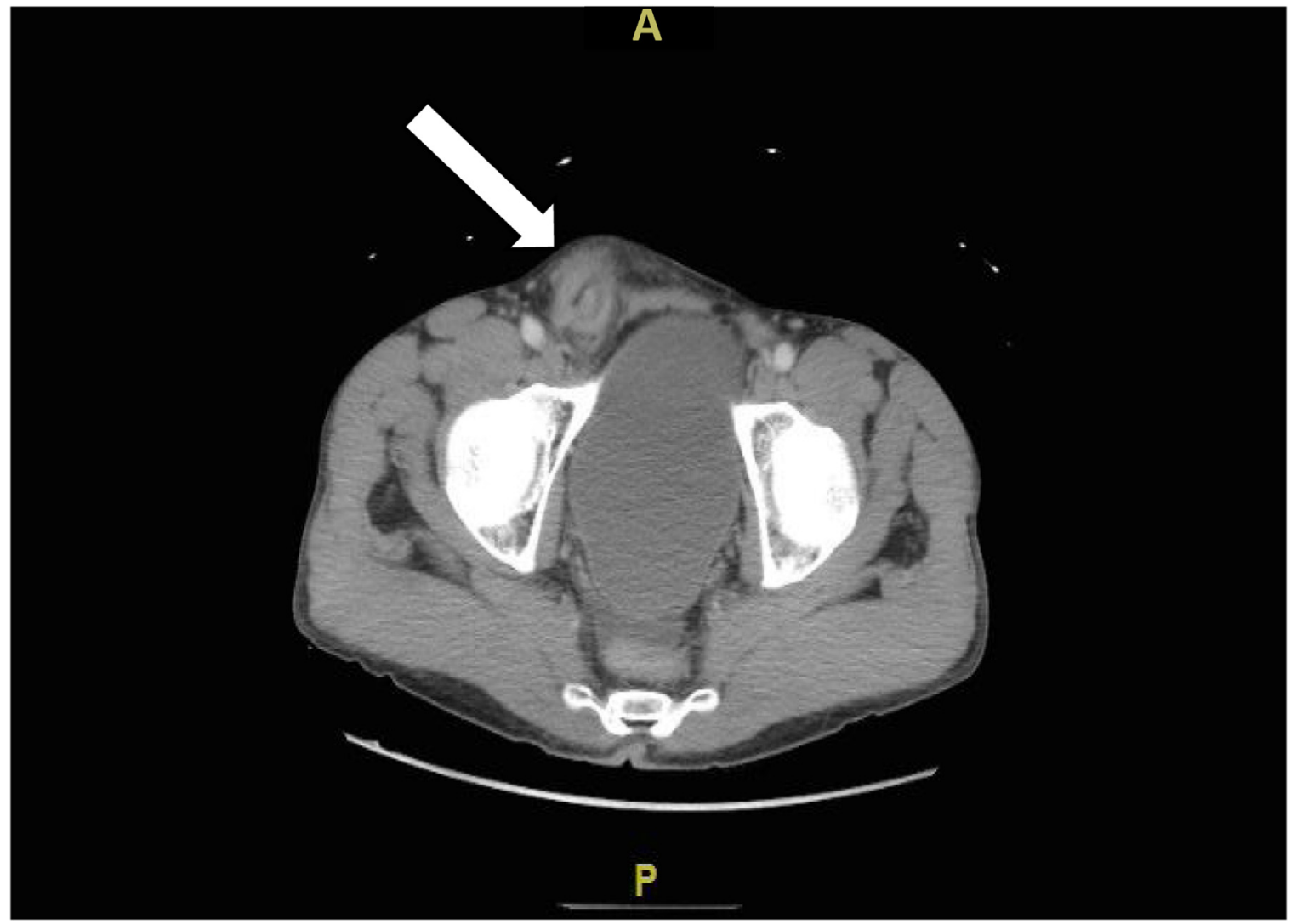

Fig. 3. CT showing right inguinal hernia - chronic inflammation and fibrosis at the inguinal hernia and sac.

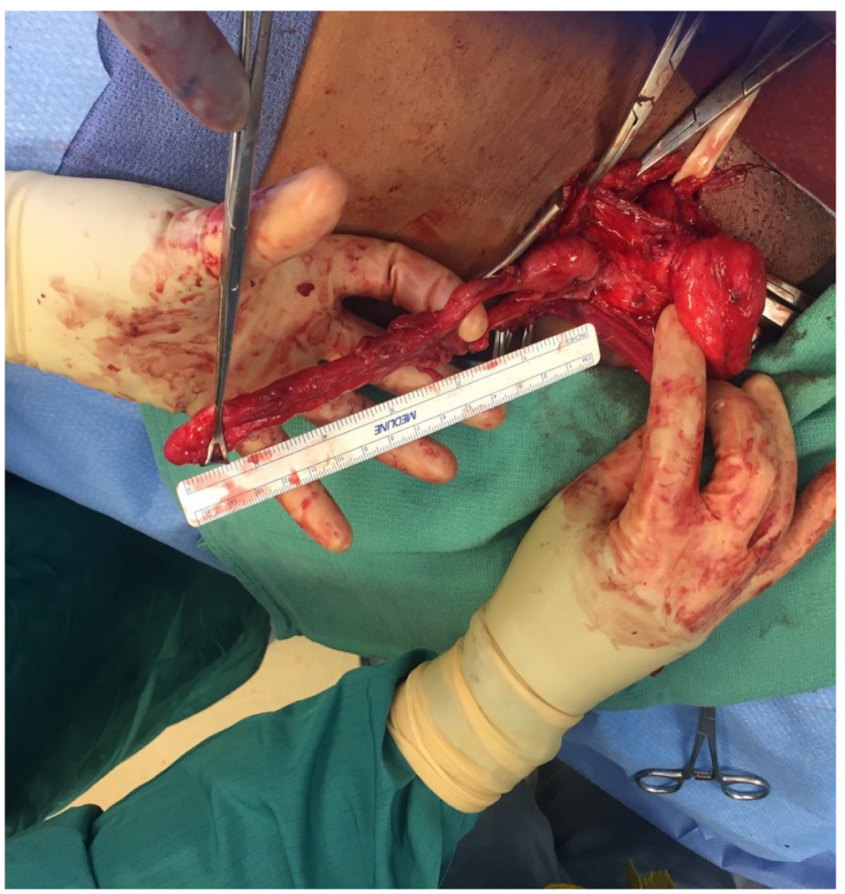

Fig. 4. Right incarcerated inguinal hernia (Amyand type 2) - opened hernia sac with an appendix measuring $15 \mathrm{~cm}$ long.

\section{Presentation of case}

A 59-year-old male with a history of a previously reducible right inguinal hernia for approximately 3 years presented to the Emer- gency Department with acute abdominal pain, right groin mass, with associated nausea and vomiting that started about $24 \mathrm{~h}$ prior to arrival. The patient reported that the hernia is usually painless 
Table 1

Losanoff and Basson classification of Amyand's hernia.

\begin{tabular}{|c|c|c|}
\hline Classification & Description & Surgical management \\
\hline Type 1 & Normal appendix in an inguinal hernia & Hernia reduction, mesh repair \\
\hline Type 2 & Acute appendicitis in an inguinal hernia, without abdominal sepsis & Appendectomy, primary repair of hernia without mesh \\
\hline Type 3 & Acute appendicitis in an inguinal hernia, with abdominal wall or peritoneal sepsis & Laparotomy, appendectomy, primary repair without mesh \\
\hline Type 4 & Acute appendicitis in an inguinal hernia, with abdominal pathology & Manage as Type $1-3$, investigate pathology as needed \\
\hline
\end{tabular}

and reducible but has recently elicited a constant sharp pain that is worse with movement.

During examination the patient was afebrile and hemodynamically stable with a high normal white count of 10.8 with a left shift. His abdominal exam was significant for distension with right lower quadrant tenderness and an obviously right inguinal mass that was tender and non-reducible. The rest of the physical exam was unremarkable. Initial computed tomography (CT) confirmed a right incarcerated inguinal hernia with herniated loops of bowel within the right inguinal region (Figs. 1-3). After discussing the risks and benefits with the patient he agreed to proceed with surgery and was taken to the operating room for an emergent right inguinal hernia exploration.

Intraoperatively, a large chronic appearing hernia was apparent with dense tissue that had to be meticulously excised to identify the cord structures. Once isolated, the hernia sac was explored and a long tubular structure was identified. The vermiform appendix was non-perforated or gangrenous however displayed mild to moderate inflammation and measured $15 \mathrm{~cm}$ with fibrinous adhesions within the hernia sac (Fig. 4). Due to the appearance of the appendix, the patient's clinical picture, and presence of adhesions, the patient underwent an appendectomy and hernia repair with polypropylene mesh with no complications. The patient received a 24-hour course of antibiotics and was discharged from the hospital on postoperative day two. The histopathology was consistent with an appendix with serosal adhesions and periappendicitis. The patient was seen for follow-up after in the office and the incision site was healing well with no signs of infection.

\section{Discussion}

The current generally accepted treatment algorithm for Amyand's hernia is essentially contingent on the appendix's condition within the hernia sac. Losanoff and Basson created a classification system for Amyand's hernia based on different conditions they identified (Table 1 ). This system identifies four unique hernia types: (1) Normal appendix in an inguinal hernia, (2) Acute appendicitis in an inguinal hernia, without abdominal sepsis, (3) Acute appendicitis in an inguinal hernia, with abdominal wall or peritoneal sepsis, and (4) Acute appendicitis in an inguinal hernia, with other abdominal pathology [4].

There is considerable agreement regarding the surgical treatments for types 3-4, which entails an appendectomy with a primary hernia repair and avoidance of mesh [5]. However, as Kose et al. points out there is current controversy regarding the surgical treatment for types 1-2 and the Losanoff and Basson's classification system with newly recognized types of Amyand's hernia not previously mentioned in their system [6]. Similar to our case, Kose et al. recognized a type of Amyand's hernia with the vermiform appendix connected to the hernia sac via fibrous bands that was unable to be freed without resecting the appendix. In our case the appendix appeared inflamed, which made it a type 2 Amyand's hernia, which necessitated an appendectomy [7,8]. According to Losanoff's classification if an appendectomy is performed then mesh should not be used due to the risk of infection. Kose et al. argues that in the situation with a healthy appearing appendix unable to be reduced (incarcerated) with no signs of inflammation an appendectomy be performed and the surgeon should then proceed with a tension free repair with mesh as the risk of infection is low compared to the risk of hernia recurrence with a primary repair only.

In our case we performed an appendectomy, and elected to proceed with a tension free hernia repair with polypropylene mesh for several reasons including the appendix condition of mild inflammation without perforation or gangrene, the patient was a generally healthy and active individual, and due to the large size of the chronic hernia the risk of recurrence with a primary repair was greater.

There are documented reports of successful outcomes for recurrent hernias identified as type 2 Amyand's hernia utilizing an appendectomy and tension free hernia repair with mesh. Velimezis et al. identified a 78-year-old man with a recurrent hernia and an inflamed non-perforated vermiform appendix that was subsequently resected and due to the recurrence necessitated a tension free hernia repair with a successful outcome and no signs of infection or recurrence up to 36 months follow up [9]. Ali et al describes three cases of type 2 Amyand's hernia, similar to our case, that was successfully treated with an appendectomy and tension free hernia repair with no surgical site infections or signs of recurrence in follow up of one month to three years Amyand's hernia, which necessitated [10].

In our case with a type 2 Aymand's hernia we performed an appendectomy with a tension free hernia repair using polypropylene mesh with a successful outcome. Although this is one report with a positive outcome in addition to the handful of similar cases mentioned, this is a testament to the fact that this rare surgical issue needs to be researched further. Without more research, surgeons will continue to make sub-optimal decisions not evidence based which potentially increases patient morbidity. More research will ultimately allow surgeons to be better equipped when dealing with this rare entity that is mostly diagnosed intraoperatively.

Based on our results along with the previously mentioned successful outcomes using mesh, it may be beneficial for patients with type 2 Amyand's hernia to have an appendectomy with a tension free hernia repair with mesh. In cases where the appendix is inflamed with no perforations or frank gangrene then, depending on other factors such overall health and hernia size, the surgeon could consider proceeding with a tension free hernia repair with mesh as the overall risk of infection may be lower than the risk of lifelong recurrence with a primary hernia repair. However, there are clear controversies regarding the recognized classification system and treatment algorithm and further evidence-based studies are essential for future successful patient outcomes.

\section{Conclusion}

We present a case report of a rare entity known as an Amyand's hernia that presented as an incarcerated hernia that was diagnosed intraoperatively with an inflamed appendix, known as type 2 that was subsequently treated with an appendectomy and tension free hernia repair with polypropylene mesh with a successful outcome. Controversy exists regarding the use of mesh in type 2 Amyand's hernia however we add to the argument of pro-mesh. However, more research is needed to provide surgeons with evidence-based 
standardized approaches for dealing with this unique situation to ensure optimal patient outcomes.

\section{Conflicts of interest}

None.

\section{Funding source}

None.

\section{Ethical approval}

Patient consent was received and ethical approved was granted by our institution's IRB committee.

\section{Consent}

Written informed consent was obtained from the patient for publication of this case report and accompanying images. A copy of the written consent is available for review by the Editor-in-Chief of this journal on request.

\section{Author contribution}

Youssef Shaban - Conception of study, acquisition of data, analysis and interpretation of data, drafting and revision of article. Dessy Boneva - Management of case, revision of article, final approval of the version to be submitted Adel Elkbuli - Acquisition of data, Analysis of data, drafting and revision of article. Mark McKenney Analysis of data, revision of article.

\section{Registration of research studies}

This is a case report study.

\section{Guarantor}

Mark McKenney, Dessy Boneva, Adel Elkbuli.

\section{References}

[1] C. Amyand, Of an inguinal rupture, with a pin in the appendix caeci, incrusted with stone, and some observations on wounds in the guts, Philos. Trans. R. Soc. Lond. 39 (1736) 329-336.

[2] Adamantios Michalinos, Demetrios Moris, Spyridon Vernadakis, Amyand's hernia: a review, Am. J. Surg. 207 (2013), http://dx.doi.org/10.1016/j.amjsurg. 2013.07.043.

[3] R.A. Agha, A.J. Fowler, A. Saetta, I. Barai, S. Rajmohan, D.P. Orgill, for the SCARE Group, The SCARE statement: consensus-based surgical case report guidelines, Int. J. Surg. 34 (2016) 180-186.

[4] J.E. Losanoff, M.D. Basson, Amyand hernia: a classification to improve management, Hernia (Jun;12 (3)) (2008) 325-326, http://dx.doi.org/10.1007/ s10029-008-0331-y, Epub 2008 Jan 24. PMID: 18214637.

[5] William Kromka, Aline S. Rau, Charles J. Fox, Amyand's hernia with acute gangrenous appendicitis and cecal perforation: a case report and review of the literature, Int. J. Surg. Case Rep. 44 (2018) 8-10, PMC. Web. 23 Mar. 2018

[6] E. Kose, A. Sisik, M. Hasbahceci, Mesh inguinal hernia repair and appendectomy in the treatment of amyand's hernia with non-inflamed appendices, Surg. Res. Pract. 2017 (2017), http://dx.doi.org/10.1155/2017/ 7696385, 7696385; Epub 2017 Jan 17.PMID: 28194430.

[7] Jonathan Green, G. Luke, Gutwein amyand's hernia: a rare inguinal hernia, J. Surg. Case Rep. 9 (September (1)) (2013), http://dx.doi.org/10.1093/jscr/ rjt043, rjt043.

[8] A. Morales-Cárdenas, C.F. Ploneda-Valencia, V.H. Sainz-Escárrega, et al., Amyand hernia: case report and review of the literature, Ann. Med. Surg. 4 (2) (2015) 113-115, http://dx.doi.org/10.1016/j.amsu.2015.03.007.

[9] G. Velimezis, N. Vassos, G. Kapoquiannatos, et al., Incarcerated recurrent inguinal hernia containing an acute appendicitis (amyand hernia): an extremely rare surgical situation, Arch. Med. Sci. (April 113(3)) (2017) 702-704, http://dx.doi.org/10.5114/aoms.2016.60403, Epub 2016 Jun 7. PMID: 28507592 PMCID: PMC5420623.

[10] S.M. Ali, K.A. Malik, H. Al-Qadhi, Amyand's hernia: study of four cases and literature review, Sultan Qaboos Univ. Med. J. (May 12(2)) (2012) 232-236, Epub 2012 Apr 9. PMID: 22548145 PMCID: PMC3327573.

This article is published Open Access at sciencedirect.com. It is distributed under the IJSCR Supplemental terms and conditions, which permits unrestricted non commercial use, distribution, and reproduction in any medium, provided the original authors and source are credited. 